\title{
Cloning and functional characterization of two cDNAs encoding NADPH- dependent 3-ketoacyl-CoA reductased from developing cotton fibers
}

\author{
Yong Mei QIN ${ }^{1,2}$, Francois MA PUJOL ${ }^{3}$, Yong Hui SHI ${ }^{1,2}$, Jian Xun FENG $^{1,2}$, Yi Ming LIU ${ }^{1,2}$, \\ Alexander J KASTANIOTIS ${ }^{3}$, J Kalervo HILTUNEN ${ }^{3}$, Yu Xian ZHU ${ }^{1,2^{*}}$ \\ ${ }^{1}$ National Laboratory of Protein Engineering and Plant Genetic Engineering, College of Life Sciences, Peking University, \\ Beijing, 100871, China. \\ ${ }^{2}$ Department of Biochemistry and Molecular Biology, College of Life Sciences, Peking University, Beijing, 100871, China. \\ ${ }^{3}$ Biocenter Oulu and Department of Biochemistry, University of Oulu, P.O. Box 3000, FIN-90014 University of Oulu, Finland.
}

\begin{abstract}
Genes encoding enzymes involved in biosynthesis of very long chain fatty acids were significantly up-regulated during early cotton fiber development. Two cDNAs, GhKCR1 and GhKCR2 encoding putative cotton 3-ketoacyl-CoA reductases that catalyze the second step in fatty acid elongation, were isolated from developing cotton fibers. GhKCR1 and 2 contain open reading frames of $963 \mathrm{bp}$ and $924 \mathrm{bp}$ encoding proteins of 320 and 307 amino acid residues, respectively. Quantatitive RT-PCR analysis showed that both these genes were highly preferentially expressed during the cotton fiber elongation period with much lower levels recovered from roots, stems and leaves. GhKCR1 and 2 showed 30\%-32\% identity to Saccharomyces cerevisiae Ybr159p at the deduced amino acid level. These cotton cDNAs were cloned and expressed in yeast haploid $y b r 159 w \Delta$ mutant that was deficient in 3-ketoacyl-CoA reductase activity. Wild-type growth rate was restored in $y b r 159 w \Delta$ cells that expressed either GhKCR1 or 2. Further analysis showed that GhKCR 1 and 2 were co-sedimented within the membranous pellet fraction after high-speed centrifugation, similar to the yeast endoplasmic reticulum marker ScKar2p. Both GhKCR(s) showed NADPH-dependent 3-ketoacyl-CoA reductase activity in an in vitro assay system using palmitoyl-CoA and malonyl-CoA as substrates. Our results suggest that GhKCR1 and 2 are functional orthologues of ScYbr159p.
\end{abstract}

Keywords: very-long-chain fatty acids, endoplasmic reticulum, fatty acid elongation system, 3-ketoacyl-CoA reductase, Gossypium hirsutum, short-chain alcohol dehydrogenase/reductase protein family.

\section{INTRODUCTION}

Cotton fibers are seed trichomes of ovule epidermis that have significant economical importance in textile production. Fiber properties such as length, strength, uniformity and yarn spinning efficiency are no longer entirely satisfactory predictors as our society progresses forward. Instead, surface friction forces that resulted directly from the chemical constituents of fiber cells play an important role in modern costume industry [1]. As derivatives of very long chain fatty acids (VLCFA, fatty

\footnotetext{
*Correspondence: Yu Xian ZHU, National Laboratory of Protein Engineering and Plant Genetic Engineering, Peking University, Beijing, 100871, China.

Fax: 86-10-6275 4427

E-mail: zhuyx@water.pku.edu.cn.
}

acids $>$ C18), waxes are major components of cotton fiber cuticle [2] and display inverse relationships with micronaire, a measure of fiber linear density and fineness [3]. Recently, it has been reported that an $\mathrm{ABC}$ transporter from Arabidopsis thialiana mediated transport of lipids out of epidermal cells to the plant surface for biosynthesis of waxy cuticle [4].

Biosynthesis of fatty acids is a primary metabolic event representing a vital aspect for every cell of all organisms to survive [5]. Plants synthesize fatty acids within the plastids, and use a discrete and highly conserved group of dissociated enzymes named fatty acid synthase system type 2 (FASII) [6]. This system, similarily to the eukaryotic mitochondrial FAS [7-10], differs from the mammalian cytosolic fatty acid synthase system type 1 (FASI) where all reactions are carried out by a single multifunctional polypeptide [11]. Fatty acids synthesized within plastid are 
exported to endoplasmic reticulum (ER) for elongation, resulting in the formation of VLCFAs that are essential cellular precursors of waxes, seed storage lipids and sphingolipids [5]. Biosynthesis of VLCFA in plants occurs on the cytosolic face of the ER membrane and is carried out by a membrane-bound fatty acid elongation system (FAE). The first reaction involves condensation of malonyl-CoA with a long chain acyl substrate producing a 3ketoacyl-CoA, catalyzed by 3-ketoacyl-CoA synthase (KCS). Subsequent reactions include reduction to 3hydroxyacyl-CoA catalyzed by 3-ketoacyl-CoA reductase (KCR) and dehydration to an enoyl-CoA by 3-hydroxyacylCoA dehydratase, followed by a second reduction catalyzed by trans-2-enoyl-CoA reductase to form the elongated acyl-CoA [12]. A number of plant KCS(s) that are all specific to saturated and monounsaturated fatty acids have been identified and partially characterized [2]. An orthologue of Saccharomyces cerevisiae trans-2-enoylCoA reductase was found in A. thialiana [13]. To date, 3hydroxyacyl-CoA dehydratase has not been identified from any sources.

3-Ketoacyl-CoA reductase encoded by $S$. cerevisiae YBR 159w catalyzes the second important step of VLCFAs biosynthesis [14, 15] and is a member of the short-chain alcohol dehydrogenase reductase family (SDR) [16]. Disruption of $S c Y B R 159 w(y b r 159 w \Delta)$ resulted in significantly reduced VLCFA synthesis and accumulation of dihydrosphignosine, phytosphingosine and ceramides [15]. Four plant KCRs including maize YBR159 (GL8) [17, 18], A. thaliana YBR159 [14] and two isoforms of Brassica napus [19] were found to be orthologues of yeast Ybr159p. The GL 8 mutant maize displayed defects in the biosynthesis of the cuticular waxes deposited on the outer epidermis, rendering the plant a characteristic "glossy" phenotype [17]. In this study, we identified two cDNAs from developing cotton fiber cells. We characterized these cotton proteins by functional complementation of a yeast mutant, $y$ br 159w $\Delta$. Results obtained from biochemical assays indicated that the cotton gene products possess NADPH dependent 3-ketoacyl-CoA reductase activities that are important for VLCFAs biosynthesis.

\section{MATERIALS AND METHODS}

\section{Library screen, sequencing and phylogenetic analyses}

Complementary DNA library was constructed by using total RNA extracted from 10 dpa cotton (Gossypium hirsutum L.cv. Xuzhou 142) fiber cells [20]. The library was screened using the AtYBR159w [14] coding sequence as a probe at low stringency as previously described [21]. Putative full-length GhKCR1 and GhKCR2 cDNAs were obtained by sequencing all colonies that hybridized to the probe from both ends.

\section{QRT-PCR analysis of $G h K C R(s)$ expression patterns}

Real time quantitative RT-PCR (QRT-PCR) was carried out us- ing the SYBR green PCR kit (Applied Biosystems) in a DNA Engine Opticon-Continuous Fluorescence Detection System (MJ Research). Following PCR primers were used: GhKCR1, 5'-CACTTTGGGTTCTTTATCACTCTT-3' (forward, F) and 5'-TTTATCTTCTTCACGCCTTCAT-3' (reverse, R); GhKCR2, 5'- TATCCTTTCTCACCCGCTCC-3' (F) and 5'- CACCACTTCCTC CTCCACCTC$3^{\prime}(\mathrm{R})$. Each pair of primers produced a single DNA fragment of appropriate size in any stages of fiber development, different cotton tissue and mutant cotton ovules. All QRT-PCRs were carried out with following parameters: initiation with a 10 min denaturation at $95^{\circ} \mathrm{C}$, followed by 42 cycles of amplification with $10 \mathrm{sec}$ denaturation at $94^{\circ} \mathrm{C}, 20 \mathrm{sec}$ annealing according to the melting temperature of each pair of primers, $20-30 \mathrm{sec}$ extension at $72^{\circ} \mathrm{C}$, then read the plate for fluorescence data collection at $78-80^{\circ} \mathrm{C}$. After a final extension at $72^{\circ} \mathrm{C}$ for $5-10 \mathrm{~min}$, melting curve was performed from $65^{\circ} \mathrm{C}$ to $95^{\circ} \mathrm{C}$ ( $1 \mathrm{sec}$ hold per $0.2^{\circ} \mathrm{C}$ increasing) to check the specificity of the amplified product. Samples were analyzed in triplicates using independent RNA samples and were quantified by the comparative cycle threshold method [22].

\section{Functional complementation of yeast ybr $159 w \Delta$ mutant strain by cotton enzymes}

ORFs of GhKCR1 and GhKCR2 were amplified using the following primers: 5'- CACCACAAAATGGAAGCCTGCTTCTTCGATACT-3' (F1) and 5'-TTCCTTCTTCCTAGAATCTTTCAT-3' (R1); 5'- CACCACAAAATGCAGCCCACATGGTTGTTAGC-3' (F2) and 5'-CGCAAGGACCTCTGCTCGCC TTCG-3' (R2). The sequence "CACC" at the beginning of each forward primer is required for cloning into $\mathrm{pENTR} \mathrm{TOPO}^{\circledR}$ vector (Invitrogen) and the sequence "ACAAA" is used to enhance gene expression in yeast. The constructed vectors, pENTR TOPO::GhKCRl and pENTR TOPO::GhKCR2 were verified by sequence analysis. The genes were transferred from $p E N T R$ TOPO to yeast expression vector $\mathrm{pYTV}$ [23] via LR reaction, using homologous sequences present on both vectors, resulting in pYTV::GhKCR1 and pYTV::GhKCR2. Gal1 promoter was fused at the N-terminus and $3 \times$ FLAG, $6 \times$ His and $2 \times \operatorname{IgG}$ were present to facilitate detection of the fusion protein [23].

The S. cerevisiae haploid strain BY4741 ybr159w (MATa; his 3 $\Delta 1$; leu $2 \Delta 0$; met15 $\Delta 0$; ura3 $\Delta 0$; ybr159w::kanMX4, EUROSCARF) was grown on YPD $(1 \%[\mathrm{wt} / \mathrm{v}]$ yeast extract, $2 \%[\mathrm{wt} / \mathrm{v}]$ peptone, and $2 \%$ [wt/v] D-glucose) supplemented with $300 \mu \mathrm{g} / \mathrm{ml}$ of Geneticine. pYTV::GhKCR(s) were transformed into ybr159w $\Delta$ mutant cells. Wild-type strain BY4741 and ybr159w $\Delta$ mutant cells were transformed with plain pYTV separately and were used as controls. The transformants were selected on synthetic complete medium lacking uracil (Sc-Ura) plates. The growth rates of wild-type BY4741 transformed with pYTV, $y b r 159 w \Delta$ mutant transformed with pYTV, and those transformed with pYTV::GhKCR(s) for overexpression in ybr159w $\Delta$ were examined on synthetic complete medium lacking tryptophan (Sc-Trp) containing $2 \%[\mathrm{wt} / \mathrm{v}]$ galactose and $0.05 \%$ [wt/v] D-glucose.

\section{Semi-quantitative RT-PCR analysis}

Total RNA was extracted from yeast cells transformed by pYTV:: $G h K C R(\mathrm{~s})$ using RNeasy Mini Kit (Qiagen) and cDNA was synthesized from $5 \mu \mathrm{g}$ total RNA using the SUPERSCRIPT ${ }^{\mathrm{TM}}$ first-strand synthesis system for RT-PCR (Invitrogen). RT-PCR primers were the same as described in the previous section for ORF cloning. Yeast actin gene, ACT1 (Accession Number NP_116614), was used as internal control in parallel reactions. 


\section{Preparation of ER extracts from yeast cells}

Yeast cells transformed by pYTV or pYTV::GhKCR(s) were grown to exponential phase $\left(2-4 \times 10^{6} \mathrm{cell} / \mathrm{s} / \mathrm{ml}\right)$ in Sc-Ura medium containing $2 \%(\mathrm{wt} / \mathrm{v})$ galactose and $0.05 \%(\mathrm{wt} / \mathrm{v}) \mathrm{D}$-glucose at $30^{\circ} \mathrm{C}$. The cells were harvested by centrifuging for $15 \mathrm{~min}$ at $1300 \mathrm{~g}, 4^{\circ} \mathrm{C}$. The cell pellets were washed with sterile $\mathrm{H}_{2} \mathrm{O}$, and suspended in icecold lysis buffer containing $50 \mathrm{mM}$ Tris, $\mathrm{pH} 7.5,1 \mathrm{mM}$ EGTA, $0.5 \mathrm{mM}$ $\beta$-mercaptoethanol, $1 \mathrm{mM}$ phenylmethlsulfonyl fluoride (PMSF) and $2 \mu \mathrm{g} / \mathrm{ml}$ pepstatin A. The cells were disrupted with glass beads and centrifuged for $15 \mathrm{~min}$ at $15000 \mathrm{~g}, 4^{\circ} \mathrm{C}$ to remove the cell debris. The supernatants were centrifuged for $90 \mathrm{~min}$ at $85,000 \mathrm{~g}$ in a Sorval Ti70 rotor at $4{ }^{\circ} \mathrm{C}$ to generate pellet (P85) and supernatant (S85) fractions. P85 was suspended in lysis buffer and used for elongase assay. The protein concentrations were determined by the method of Lowry et al [24], using bovine serum albumin as the standard. Equivalent amount of total lysate, P85 and S85 were precipitated by adding trichloroacetic acid (TCA) to $10 \%$ final concentration and processed for immunoblotting.

\section{Immunoblotting}

Equivalent amount of total lysate, high speed supernatant (S85) and membrane pellet (P85) were separated on 12\% SDS-PAGE and electro-transferred to PVDF Hybond ${ }^{\mathrm{P}}$ membrane (Amersham Biosciences). Recombinant GhKCR(s) were detected by using mouse monoclonal antibody against His-tag as the primary antibody (Invitrogen) and affinity-purified goat anti-mouse IgG conjugated with horseradish peroxidase as the second antibody. S. cerevisiae ER marker protein Kar2p [25] was detected by using polyclonal rabbit antibody against ScKar2p [26], a gift from M. Rose, as the primary antibody and affinity-purified goat anti-rabbit IgG conjugated with horseradish peroxidase as the second antibody (Bio-Rad Laboratories). The recognized epitopes were detected by using the ECL Western blotting detection system (Amersham Biosciences).

\section{Elongase assays of cotton 3-ketoacyl-CoA reductases over- expressed in yeast cells}

Palmitoyl-CoA was used as acyl-CoA acceptor in all reactions. The elongation assays were divided into two groups. One was supplemented with both NADPH and NADH [15], and another was supplemented only with NADH. The elongation assays contained $50 \mathrm{mM}$ Tris- $\mathrm{HCl}$, pH 7.5, $1 \mathrm{mM} \mathrm{MgCl} 2,0.15 \mathrm{mM}$ Triton X-100, $1 \mathrm{mM}$ NADPH, $1 \mathrm{mM}$ NADH, $10 \mathrm{mM} \beta$-mercaptoethanol, $40 \mu \mathrm{M}$ palmitoyl-CoA, $60 \mu \mathrm{M}\left[2-{ }^{14} \mathrm{C}\right]$ malonyl-CoA $(6.5 \mathrm{dpm} / \mathrm{pmol}$, PerkinElmer Life Sciences) in a final reaction volume of $0.2 \mathrm{ml}$. To initiate the elongation reaction, $0.05 \mathrm{mg}$ ER protein extracted from yeast cells was added. The reaction was incubated at $30^{\circ} \mathrm{C}$ and continued for the indicated time. The reactions were stopped by adding $0.1 \mathrm{ml}$ of $75 \% \mathrm{KOH} \mathrm{(w/v)} \mathrm{and} 0.2 \mathrm{ml}$ of ethanol, saponified at $70^{\circ} \mathrm{C}$ for $1 \mathrm{~h}$, and then acidified by adding $0.4 \mathrm{ml}$ of $5 \mathrm{~N} \mathrm{HCl}$ with $0.2 \mathrm{ml}$ of ethanol. Fatty acids were collected using $1 \mathrm{ml}$ of hexane. The extractions were dried under nitrogen, and separated by thin-layer chromatography (TLC) using hexane/diethyl ether/acetic acid (30:70:1). The TLC plates were exposed to a PhosphorImager screen, the resulting image was analyzed, and the lipids were quantified using a BioImaging Analyzer with 2D Image master software (Amersham Biosciences).

\footnotetext{
Hydropathy analysis

Hydropathy plot for each GhKCR gene was produced according to the method described previously[27].
}

\section{RESULTS}

Identification of two cotton 3-ketoacyl-CoA reductases

A total of 19 colonies were obtained as a result of library screen with 15 found to encode for GhKCRl and 4 for GhKCR2 after sequencing of the full-length insert. GhKCR 1 possessed a 963 bp ORF that encoded a protein of 320 amino acids with a predicted molecular mass of 36 kD. GhKCR2 contained a 924 bp ORF that encoded a protein of 307 amino acids with a predicted molecular mass of $34 \mathrm{kD}$. We submitted GhKCRl and 2 to GenBank with accession numbers AY902466 and AY902467, respectively. As shown in Fig. 1, GhKCR1 shared $68 \%$ sequence identity to that of AtYBR159 and Bn-KCR2, 66.7\% to BnKCR1, $65 \%$ to ZmYBR 159 , 30\% to ScYbr159p, 39\% to human HsKCR and $41 \%$ to mouse MuKCR. GhKCR2 shared 59\% total sequence identity with that of GhKCR1 and showed similar or slightly lower amino acid identities to the abovementioned enzymes. All these proteins belong to SDR family [16] characterized by the presence of Rossmann fold (the nucleotide binding site) and a triad of catalytically important and highly conserved Ser-Tyr-Lys residues. A canonical dilysine ER retention signal is present in the C-terminus of GhKCR1 but not in GhKCR2. These results suggested that GhKCR1 and 2 might be the orthologues of AtYBR159.

\section{GhKCR(s) showed fiber-preferential expression pat- terns}

Relative transcript levels of $G h K C R(\mathrm{~s})$ from 3, 5, 10, 15,20 dpa wild-type cotton ovules together with their fibers (wt) were compared with either those of $0 \mathrm{dpa} w \mathrm{t}$ ovules or 10 dpa ovules of a fuzzless-lintless mutant $(f l)$ by QRT-PCR analysis (Fig. 2). GhKCR1 transcripts increased about 30-fold and that of GhKCR2 increased about 20 -fold only $5 \mathrm{~d}$ after flowering, whereas, very low levels of transcripts were observed in roots, stems and leaves for either gene (Fig. 2). Both GhKCRs were suppressed significantly in ovules of the $f l$ mutant as well (Fig. 2).

\section{Functional complementation of yeast mutant ybr159w $\Delta$ cells by the cotton enzymes}

As seen in Fig. 3A, without transformation of GhKCR (s), S. cerevisiae haploid ybr159w $\Delta$ mutant cells grew very slowly on Sc-Trp plate. Wild-type growth rate was resumed in mutant cells expressing either $G h K C R 1$ or 2 suggesting that heterologous expression of $G h K C R(\mathrm{~s})$ was able to complement the genetic deficiency. In agreement with the plate assay, ybr159w $\Delta$ mutant cells transformed by pYTV:: GhKCR2 performed similarly with that of wild-type whereas cells expressing $G h K C R 1$ grew even better than the wild-type (Fig. 3B). RT-PCR analysis of ybr $159 \mathrm{w} \Delta$ mutant cells transformed by either pYTV::GhKCRl or 


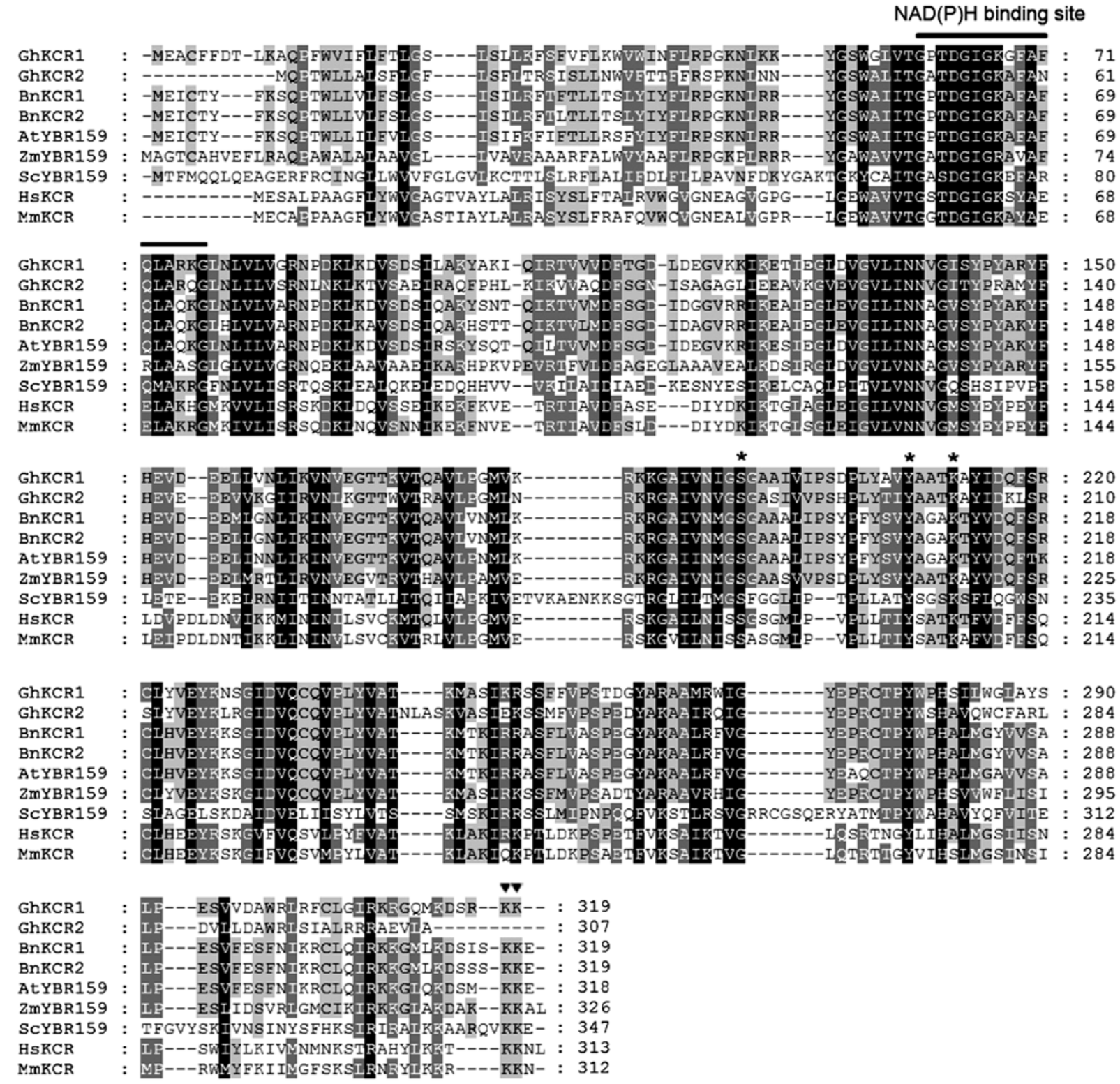

Fig. 1 Amino acid sequence alignment of GhKCR1 and GhKCR2. Amino acid sequence alignment of GhKCR1 (Genbank ${ }^{\mathrm{TM}}$ accession no. AY902466) and GhKCR2 (Genbank ${ }^{\mathrm{TM}}$ accession no. AY902467) with orthologues from B. napus (Genbank ${ }^{\mathrm{TM}}$ accession no. AAO43448 and

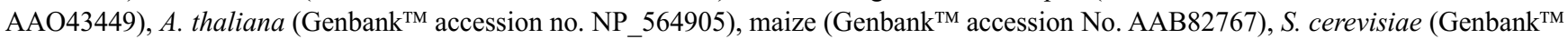
accession no. AAS56194), humans (Genbank ${ }^{\mathrm{TM}}$ accession no. AAP36605) and mouse (Genbank ${ }^{\mathrm{TM}}$ accession No. NM_00829). Black shading indicates strictly conserved residues, whereas gray shading marks regions of less strict conservation. An active triad of highly conserved S-Y$\mathrm{K}$ residues is marked with an asterisk and canonical dilysine ER retention motif is indicated $(\boldsymbol{\nabla})$.

pYTV::GhKCR2 showed that all the transgenes were actively transcribed during the log phase of yeast culture (Fig. 3C). Our data suggested that the genetic complementation phenomenon was related to the biochemical functions of individual $G h K C R(\mathrm{~s})$.

\section{Subcellular localization of GhKCR proteins expressed} in yeast

Insoluble membranous fractions (P85) were separated from the high speed supernatant (S85) by differential centrifugation using total proteins extracted from $y b r 159 w \Delta$ 

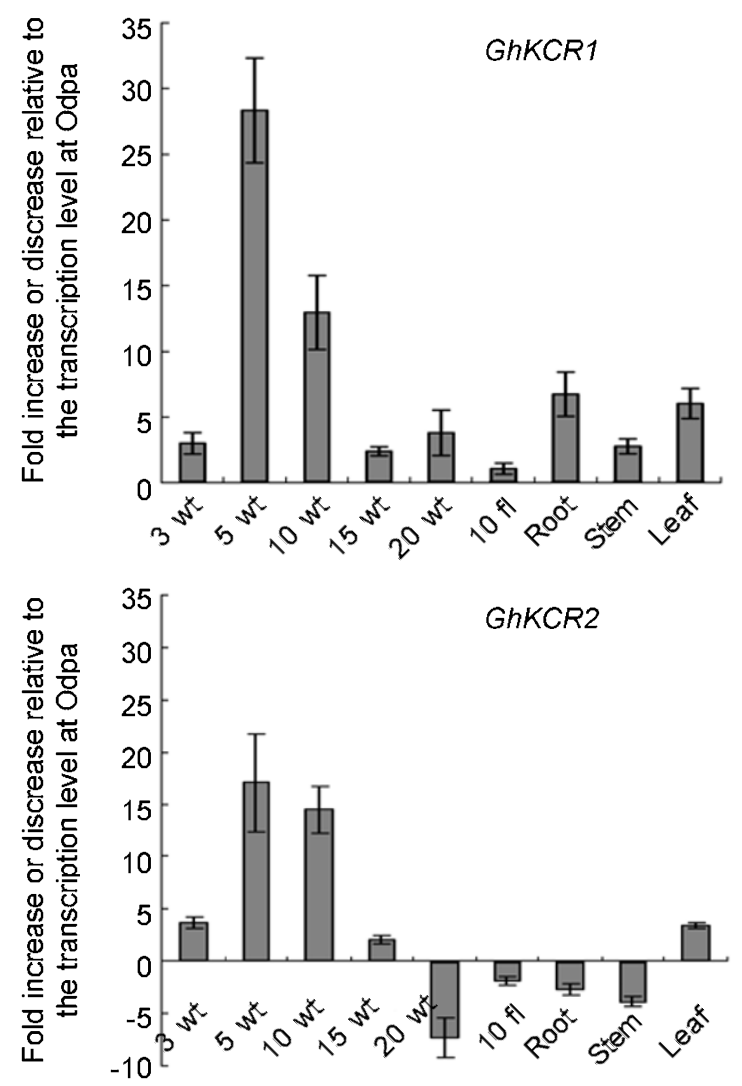

Fig. 2 Both $G h K C R$ genes are highly preferentially expressed in fast-elongating wild-type cotton fibers, but not in roots, stems, leaves and mutant cotton ovules. $0,+3,+5,+10,+15,+20$ and $+10 f l$ indicate that total RNA samples prepared from $0,3,5,10,15,20$ dpa wide-type or $10 \mathrm{dpa} f l$ mutant cotton ovules were used as the template for QRT-PCR analysis. Error bars indicate means \pm SE obtained from three independent experiments. Cotton ubiquitin gene, UBQ7 (Genbank ${ }^{\mathrm{TM}}$ accession no. AY189972), was included as the template control. Fold increase or decrease was calculated using data obtained from mRNA samples prepared from ovules harvested around the day of anthesis ( $0 \mathrm{dpa})$.

cells transformed either with pYTV or pYTV::GhKCR(s). Equivalent amount of total lysate, P85 and S85 were separated on SDS-PAGE and analyzed by immunoblotting using anti-His and anti-ScKar2p separately. Both GhKCR1 (Fig. 4A) and GhKCR2 (Fig. 4B) were detected mainly in P85 fraction in a similar manner with that of ER marker ScKar2p $[25,26]$. These data agreed with predictions of hydropathy plots since both GhKCR1 and 2 showed at least one membrane-spanning domain at their $\mathrm{N}$-termini (Fig. 4). In the total lysate (TL) and P85 fractions prepared from $y b r 159 w \Delta$ cells without being transformed by the

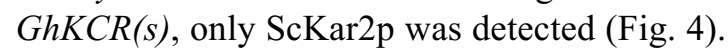

A

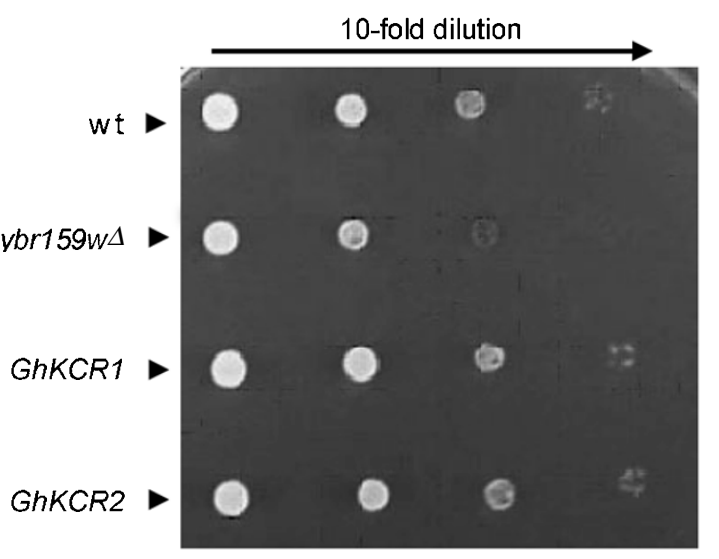

B

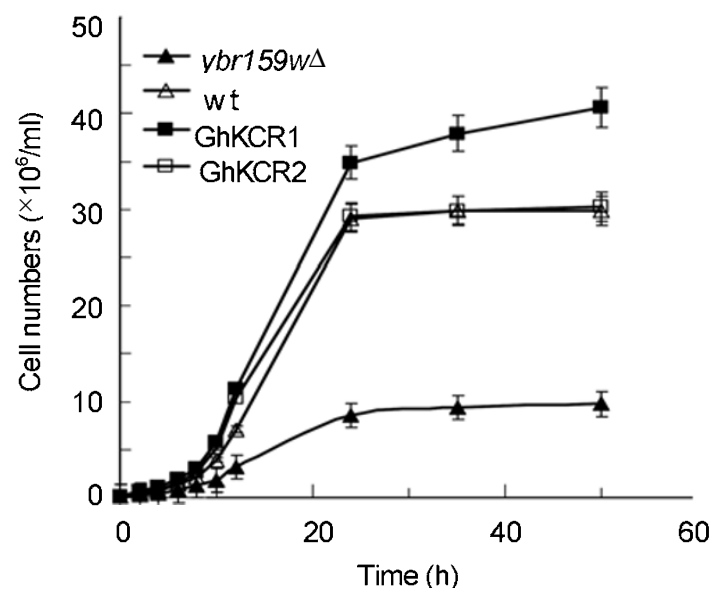

C

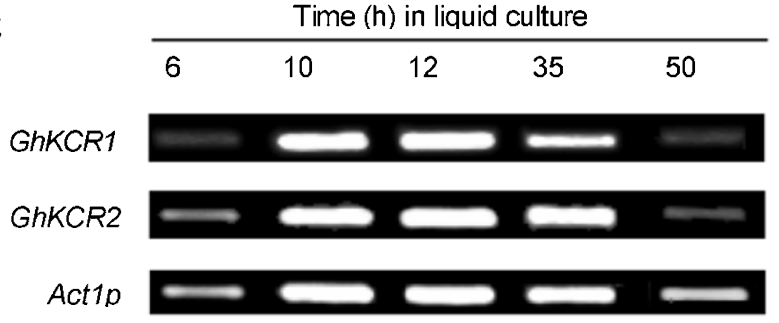

Fig. 3 Yeast $y b r 159 w \Delta$ mutant cells can be functionally complemented by overexpression of individual $G h K C R(\mathrm{~s})$. (A) Wild-type rate of cell division was obtained in $y b r 159 w \Delta$ mutant cells that overexpressed either $G h K C R 1$ or $G h K C R 2$. All cells were inoculated from overnight culture and were grown in SC-Trp , 2\% Galactose liquid media to an $\mathrm{OD}_{600}$ of 0.5 before being plated out on SC-Trp solid media in a series of 10 -fold dilutions with sterile $\mathrm{H}_{2} \mathrm{O}$. Cells were then incubated at $30^{\circ} \mathrm{C}$ for 2 days before being examined with naked eyes. (B) Growth curves of individual yeast strains cultured in SC-Trp, $2 \%$ Galactose liquid media up to $50 \mathrm{~h}$ at $30^{\circ} \mathrm{C}$. Cell numbers were determined by using a haemocytometer. Error bars indicate means \pm SE from three independent cultures. (C) RT-PCR analysis of yeast cell lines expressing different $G h K C R(\mathrm{~s})$ harvested at the indicated time after liquid culture. We included only one actin gene in the figure as the template control since it was expressed in a very similar manner in all strains. 
A

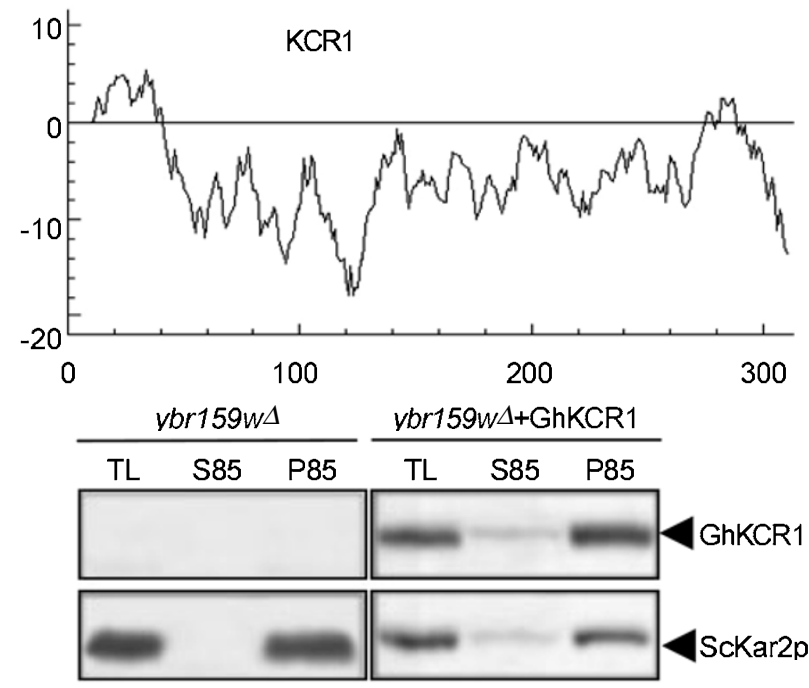

B

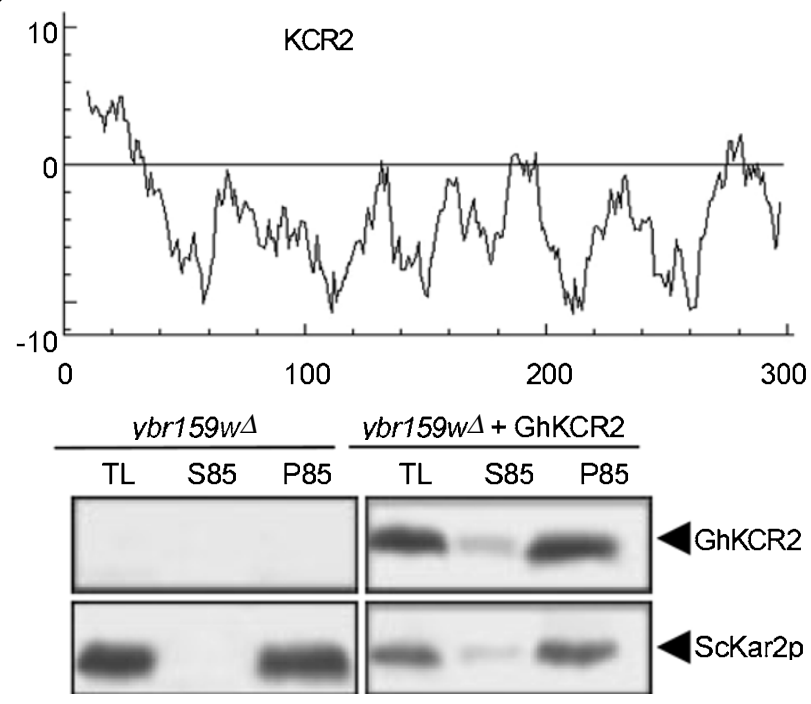

Fig. 4 GhKCRs expressed in yeast were associated with their ER fractions. Membrane-spanning topology for each GhKCR was plotted according to White and Wimley [27] and was placed in the upper panel of each subset labeled (A) and (B) representing analysis of GhKCR1 and 2, respectively. Western blotting results were reported in the lower panel of each subset. Data on the left were obtained by using protein extracted from $y b r 159 w \Delta$ mutant cells transformed by the plain vector pYTV and data on the right were obtained by using protein extracted from $y b r 159 w \Delta$ mutant cells transformed by pYTV::GhKCR1 and pYTV::GhKCR2 accordingly.

a significant amount of 3-ketostearate with a concomitant reduction in 3-hydroxystearate as well as the final elongated product, stearate (Fig. 5). Overexpression of GhKCR 1 and 2 separately in $y b r 159 w \Delta$ mutant cells in the presence of NADH and NADPH resulted in enhanced production of 3-hydroxystearate, trans-2-stearate and stearate (Fig. 5B, Tab. 1) in the assay. In the absence of NADPH as a cofactor, however, no obvious differences in the amount of intermediates and the final product generated using extract from $y b r 159 w \Delta$ mutant with or without being transformed by $G h K C R(\mathrm{~s})$ were observed (Fig. 5C, Tab.1). When both NADH and NADPH were omitted, the reactions were stopped at the intermediate of 3-keto-stearate, indicating that the reduction step was cofactor-dependent (Fig. 5D). These data suggested that the both GhKCR(s) are NADPH-dependent 3-ketoacyl-CoA reductases.

\section{DISCUSSION}

In this report, we isolated two cDNAs, GhKCRl and 2 from developing cotton fiber cells, and characterized their functions both in genetic as well as biochemical terms. The proteins were found to restore the growth rate of $S$. cerevisiae ybr159w $\Delta$ mutant to that of wild-type cells in a genetic complementation assay (Fig. 3). Recombinant GhKCR(s) displayed 3-ketoacyl-CoA reductase activities in an in vitro reaction that used palmitoyl-CoA as the substrate in the presence of malonyl-CoA (Fig. 5). Further studies showed that recombinant GhKCR1 and 2 were co-localized with the ER marker protein ScKar2p in $S$. cerevisiae mutant ybr 159w $\Delta$ cells (Fig. 4), indicating that these cotton enzymes function as microsomal 3-ketoacylCoA reductases.

In S. cerevisiae, Ybr159p was shown to constitute majority of the KCR activity since extracts from $y b r 159 w \Delta$ mutant cells leads to a significant accumulation of 3ketostearate in the elongase assay (Fig. 5B). Using extracts from both wild-type and mutant cells that overexpressed $G h K C R(\mathrm{~s}), 3-$ ketostearate was not accumulated. Instead, production of 3-hydroxystearate and stearate was enhanced. Similar levels of 3-hydroxy-fatty acyl intermediates were found using extracts from mutant cells and those that overexpressed $G h K C R(\mathrm{~s})$ when NADPH was removed in the assay system (Fig. 5C) suggesting that GhKCR(s) were NADPH-dependent 3-ketoacyl-CoA reductases. NADPH-dependent 3-ketoreductase activities were previously detected from rat liver [28]. The cofactor requirement for the mammalian microsomal KCR protein has not been settled yet $[29,30]$. The plant 3-ketoacy[acyl carrier protein] reductase involved in plastid fatty acid synthesis was NADPH-dependent [31]. NADPH binding was found to induce a conformational change that put three conserved amino acid residues into the active site to facilitate catalytic function [32].

In maize and Arabidopsis, only one gene homologous to yeast $Y B R 159 w$ was identified $[14,17]$. Recently, two highly homologous $B n K C R(\mathrm{~s})$ from Brassica genomes were 
A

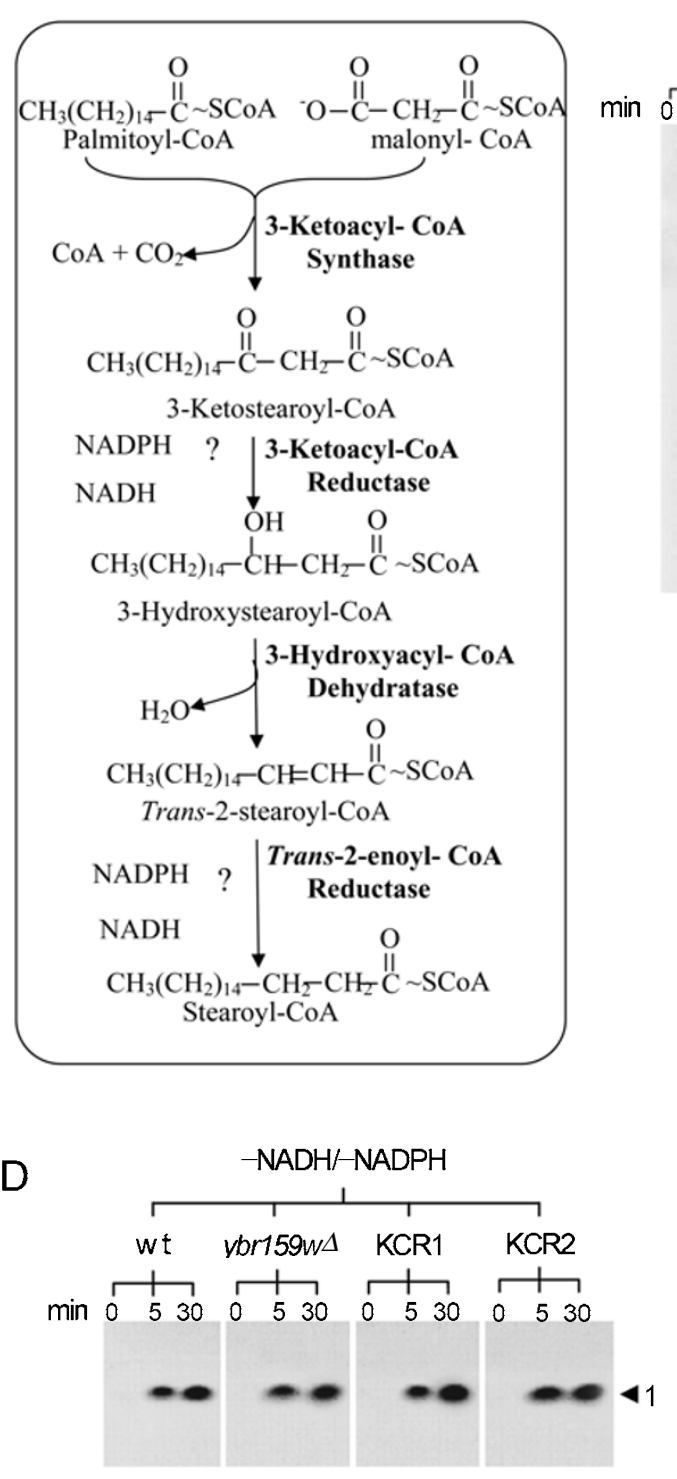

B

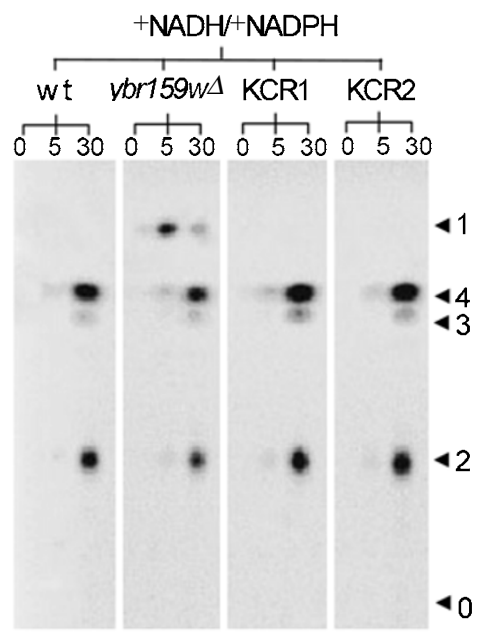

C

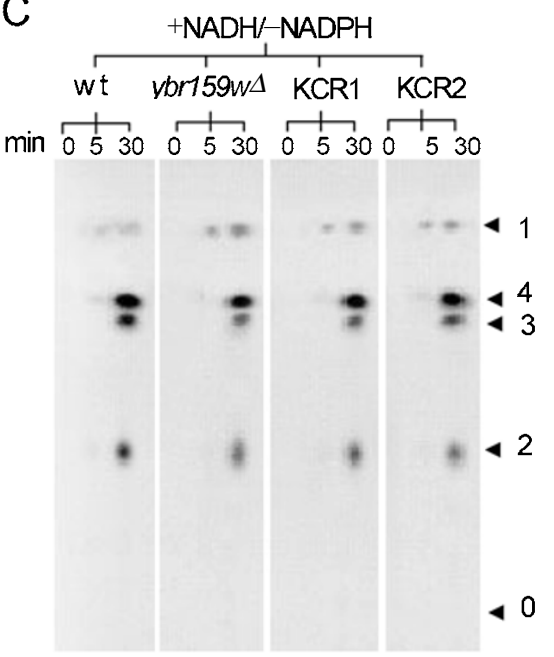

Fig. 5 GhKCRs function as NADPH-dependent 3-ketoacyl-CoA reductase in $y b r 159 w \Delta$ mutant cells. (A) Schematic drawings of reactions from palmitoyl-CoA to stearoyl-CoA. (B) ybr159w $\Delta$ mutant yeast cells transformed with GhKCRl, 2 or 3 metabolized palmitoyl-CoA at the same rate as wild-type cells in the presence of both NADH and NADPH. (C) When NADPH was not included in the experiment, all cells metabolized palmitoylCoA very slowly in the same way as that of $y b r 159 w \Delta$ mutant. (D) 3ketostearate was accumulated similarly in all cell lines if both NADPH and NADH were not added in the assay. All reactions were initiated by adding $50 \mu \mathrm{g}$ of proteins extracted from wild-type (wt), $y b r 159 w \Delta$ or $y b r 159 w \Delta$ mutant cells expressing $G h K C R 1$ or 2 (KCR1 or 2 respectively). For TLC analysis, the fatty acyl-CoA thioesters were extracted as free fatty acid. The assays were stopped at the indicated time and the fatty acids were extracted and separated by TLC. Positions labeled as 1, 2, 3, 4 and $O$ denote 3ketostearate, 3-hydroxystearate, trans-2-stearate, stearate and the origin of TLC respectively. Relative positions were determined by comparing the mobility with authentic standards as described [14] identified [19] and they are homologues of AtYBR159. Three independent lines of evidence suggest that GhKCRI and $2 \mathrm{cDNAs}$ reported in the current work might represent two different $K C R$ genes: (i) The GhKCRs show different expression patterns during various stages of cotton fiber development and also in different cotton tissue (Fig. 2). The expression profiles of the both genes are in accordance with our previous findings that several GhKCS genes encoding putative 3-ketoacyl-CoA synthases [33] and cotton genes encoding lipid transfer proteins $[34,35]$ were highly expressed at the same period. Our data indicate that long chain fatty acid biosynthesis and supply may be in high demand during the fast cell elongation period. (ii)
GhKCR1 and 2 show significant sequence similarity to ScYbr159p and share 59\% amino acid identity with each other. (iii) Although GhKCR2 protein is recovered from the microsomal fractions in the same way as GhKCR1, it is different from GhKCR1 since it lacks a predicted ER retention signal (Fig. 4).

In conclusion, we isolated two cDNAs encoding 3ketoacyl-CoA reductases from developing cotton fibers. Genetic complementation and biochemical characterization of these cotton genes in yeast cells demonstrated that they could function as NADPH-dependent 3-ketoacyl-CoA reductases. Both theoretical and experimental analysis strongly suggested that GhKCRs are involved in ER-asso- 
Tab. 1 Relative amount of 3-hydroxystearate, trans-2-stearate, and stearate of palmitate elongation catalyzed by GhKCR(s) in the presence or absence of NADPH. The values represent the relative amount of different intermediates at 30 min in relation to that measured in the yeast mutant cells transformed with plain vector that was arbitrarily defined as 1 .

\begin{tabular}{|c|c|c|c|c|c|c|c|}
\hline \multirow{3}{*}{ Products } & \multirow{3}{*}{ Steps } & \multicolumn{6}{|c|}{ Sample } \\
\hline & & \multicolumn{2}{|c|}{$\mathrm{wt}$} & \multicolumn{2}{|c|}{ GhKCR1 } & \multicolumn{2}{|c|}{ GhKCR2 } \\
\hline & & + & - & + & - & + & - \\
\hline 3-Ketostearate & 1 & & $0.56 \pm 0.09$ & & $0.85 \pm 0.1$ & & $0.85 \pm 0.08$ \\
\hline 3-Hydroxystearate & 2 & $1.3 \pm 0.1$ & $1.6 \pm 0.12$ & $1.7 \pm 0.11$ & $0.95 \pm 0.08$ & $1.5 \pm 0.09$ & $1.00 \pm 0.08$ \\
\hline Trans-2-stearate & 3 & $1.2 \pm 0.09$ & $1.9 \pm 0.1$ & $2.5 \pm 0.2$ & $1.00 \pm 0.09$ & $1.7 \pm 0.11$ & $1.10 \pm 0.09$ \\
\hline Stearate & 4 & $1.3 \pm 0.09$ & $1.9 \pm 0.14$ & $2.3 \pm 0.15$ & $1.00 \pm 0.03$ & $1.8 \pm 0.13$ & $1.00 \pm 0.09$ \\
\hline
\end{tabular}

+ , with both NADH and NADPH; -, with only NADH.

ciated very long chain fatty acid elongation. Our work indicates that the VLCFA elongation pathway is upregulated during cotton fiber development and is similar to that found in yeast cells. Whether $\operatorname{GhKCR}(\mathrm{s})$ are essential for the elongation of VLCFAs in cotton need further investigation.

\section{ACKNOWLEDGMENTS}

This work was supported by grants from China National Basic Research Program (NO. 2004CB117302), National Natural Science Foundation of China (No. 30470171), the Sigrid Jusélius Foundation Finland and the Academy of Finland. We thank Dr. Mark D. Rose for providing the ScKar2p antibody. We would also like to thank Liu D, Song WQ, Mei WQ, Lei J, Hu CY and He XC for their technical assistance.

Received, Apr 5, 2005

Revised, May 20, 2005

Accepted, May 21, 2005

\section{REFERENCES}

1 Cui XL, Price JB, Calamari TA, Hemstreet JM. Cotton wax and its relationship with fiber and yarn properties, Part I: Wax Content and Fiber Properties. Textilte Res J 2002; 72:399-404.

2 Kunst L, Samuels AL. Biosynthesis and secretion of plant cuticular wax. Prog Lipid Res 2003; 42:51-80.

3 Gamble GR. Variation in surface chemical constituents of cotton (Gossypium hirsutum) fiber as a function of maturity. J Agric Food Chem 2003; 51:7995-8.

4 Pighin JA, Zheng H, Balakshin LJ, et al. Plant cuticular lipid export requires an ABC transporter. Science 2004; 306: 702-4.

5 Ohlrogge J, Browse J. Lipid biosynthesis. Plant Cell 1995, 7:95770.

6 Lu YJ, Zhang YM, Rock CO. Product diversity and regulation of type II fatty acid synthases. Biochem Cell Biol 2004; 82:14555.

7 Harington A, Herbert CJ, Tung B, Getz GS, Slonimski PP. Identification of a new nuclear gene (CEM1) encoding a protein homologous to a $\beta$-keto-acyl synthase which is essential for mito- chondrial respiration in Saccharomyces cerevisiae. Mol Microbiol 1993; 9:545-55.

8 Schneider R, Brors B, Burger F, Camrath S, Weiss H. Two genes of the putative mitochondrial fatty acid synthase in the genome of Saccharomyces cerevisiae. Curr Genet 1997; 32:384-88.

9 Torkko JM, Koivuranta KT, Miinalainen IJ, et al. Candida tropicalis Etr1p and Saccharomyces cerevisiae Ybr026p (Mrfl'p), 2-enoyl thioester reductases essential for mitochondrial respiratory competence. Mol Cell Biol 2001; 21:6243-53.

10 Kastaniotis AJ, Autio KJ, Sormunen RT, Hiltunen JK. Htd2p/ Yhr067p is a yeast 3-hydroxyacyl-ACP dehydratase essential for mitochondrial function and morphology. Mol Microbiol 2004; 53:1407-21.

11 Smith $\mathrm{S}$. The animal fatty acid synthase: one gene, one polypeptide, seven enzymes. FASEB J 1994; 8: 1248-59.

12 Cinti DL, Cook L, Nagi MN, Suneja SK. The fatty acid chain elongation system of mammalian endoplasmic reticulum. Prog Lipid Res 1992; 31:1-51

13 Gable K, Garton S, Napier JA, Dunn TM. Functional characterization of the Arabidopsis thaliana orthologue of Tsc13p, the enoyl reductase of the yeast microsomal fatty acid elongating system. J Exp Bot 2004; 55:543-45.

14 Beaudoin F, Gable K, Sayanova O, Dunn T, Napier JA. A Saccharomyces cerevisiae gene required for heterologous fatty acid elongase activity encodes a microsomal b-keto-reductase. J Biol Chem 2002; 277:11481-8.

15 Han G, Gable K, Kohlwein SD, et al. The Saccharomyces cerevisiae YBR159w gene encodes the 3-ketoreductase of the microsomal fatty acid elongase. J Biol Chem 2002; 277:354409.

16 Persson B, Kallberg Y, Oppermann U, Jornvall H. Coenzymebased functional assignments of short-chain dehydrogenase/reductase (SDRs). Chem Biol Interact 2003; 143-144:271-8.

$17 \mathrm{Xu}$ X, Dietrich CR, Delledonne M, et al. Sequence analysis of the cloned glossy 8 gene of maize suggests that it may code for a b-ketoacyl reductase required for the biosynthesis of cuticular waxes. Plant Physiol 1997; 115:501-10.

$18 \mathrm{Xu}$ X, Dietrich CR, Lessire R, Nikolau BJ, Schnable PS. The endoplasmic reticulum-associated maize GL8 protein is a component of the acyl-coenzyme A elongase involved in the production of cuticular waxes. Plant Physiol 2002; 128:924-34.

19 Puyaubert J, Dieryck W, Costaglioli P, et al. Temporal gene 
expression of 3-ketoacyl-CoA reductase is different in high and in low erucic acid Brassica napus cultivars during seed development. Biochim Biophys Acta 2005; 1687:152-63.

$20 \mathrm{Lu} \mathrm{YC}$. Large scale cloning and characterization of fiber-specific genes through high throughput analysis. $\mathrm{PhD}$ thesis, 2002, Peking University. (In Chinese)

21 Li HY, Guo ZF, Zhu YX. Molecular cloning and analysis of a pea cDNA that is expressed in darkness and very rapidly induced by gibberellic acid. Mol Gen Genet 1998; 259:393-7.

22 Wittwer CT, Herrmann MG, Moss AA, Rasmussen RP. Continuous fluorescence monitoring of rapid cycle DNA amplification. Biotechniques. 1997; 22:130-1, 134-8.

23 Gong W, Shen YP, Ma LG, et al. Genome-wide ORFeome cloning and analysis of Arabidopsis transcription factor genes. Plant Physiol 2004; 135:773-82.

24 Lowry OH, Rosebrough NJ, Farr AL, Randall RJ. Protein measurement with the Folin phenol reagent. J Biol Chem 1951; 193: 265-75.

25 Beh CT, Rose MD. Two redundant systems maintain levels of resident proteins within the yeast endoplasmic reticulum. Proc Natl Acad Sci USA 1995; 92:9820-3.

26 Rose MD, Misra LM, Vogel JP. KAR2, a karyogamy gene, is the yeast homolog of the mammalian BiP/GRP78 gene. Cell 1989; 57:1211-21.

27 White SH, Wimley WC. Membrane protein folding and stability: physical principles. Annu Rev Biophys Biomol Struct 1999; 28: 319-65.

28 Nagi MN, Cook L, Suneja SK, et al. Evidence for two separate $\beta$ - ketoacyl CoA reductase components of the hepatic microsomal fatty acid chain elongation system in the rat. Biochem Biophys Res Commun 1989; 165:1428-34.

29 Moon YA, Shah NA, Mohapatra S, Warrington JA, Horton JD. Identification of a mammalian long chain fatty acyl elongase regulated by sterol regulatory element-binding proteins. J Biol Chem 2001; 276:45358-66.

30 Moon YA, Horton JD. Identification of two mammalian reductases involved in the two-carbon fatty acyl elongation cascade. J Biol Chem 2003; 278:7335-43.

31 Fisher M, Kroon JT, Martindale W. The X-ray structure of Brassica napus $\beta$-keto acyl carrier protein reductase and its implications for substrate binding and catalysis. Structure Fold Des 2000; 8:339-47.

32 Price AC, Zhang YM, Rock CO, White SW. Structure of $\beta$ ketoacyl-[acyl carrier protein] reductase from Escherichia coli: negative cooperativity and its structural basis. Biochemistry 2001; 40:12772-81.

33 Ji SJ, Lu YC, Feng JX, et al. Isolation and analyses of genes preferentially expressed during early cotton fiber development by substractive PCR and cDNA array. Nucleic Acids Res 2003; 31:2534-43.

34 Feng JX, Ji SJ, Shi YH, et al. Analysis of five differentially expressed gene families in fast elongating cotton fiber. Acta Biochim Biophys Sin 2004; 36:51-6.

35 Ma DP, Tan H, Si Y, Creech RG, Jenkins JN. Differential expression of a lipid transfer protein gene in cotton fiber. Biochim Biophys Acta 1995; 1257:81-4. 